Article

\title{
Constraints on Microscopic and Phenomenological Equations of State of Dense Matter from GW170817
}

\author{
Domenico Logoteta ${ }^{1,2}, * \mathbb{C}$, Ignazio Bombaci ${ }^{1,2}$ (1) \\ 1 Dipartimento di Fisica, Universitá di Pisa, Largo Bruno Pontecorvo 3, I-56127 Pisa, Italy; \\ ignazio.bombaci@unipi.it \\ 2 INFN, Sezione di Pisa, Largo Bruno Pontecorvo 3, I-56127 Pisa, Italy \\ * Correspondence: domenico.logoteta@pi.infn.it
}

Received: 23 July 2019; Accepted: 23 September 2019; Published: 25 September 2019

\begin{abstract}
We discuss the constraints on the equation of state (EOS) of neutron star matter obtained by the data analysis of the neutron star-neutron star merger in the event GW170807. To this scope, we consider two recent microscopic EOS models computed starting from two-body and three-body nuclear interactions derived using chiral perturbation theory. For comparison, we also use three representative phenomenological EOS models derived within the relativistic mean field approach. For each model, we determine the $\beta$-stable EOS and then the corresponding neutron star structure by solving the equations of hydrostatic equilibrium in general relativity. In addition, we calculate the tidal deformability parameters for the two neutron stars and discuss the results of our calculations in connection with the constraints obtained from the gravitational wave signal in GW170817. We find that the tidal deformabilities and radii for the binary's component neutron stars in GW170817, calculated using a recent microscopic EOS model proposed by the present authors, are in very good agreement with those derived by gravitational waves data.
\end{abstract}

Keywords: neutron stars; neutron star mergers; gravitational waves; equation of state

\section{Introduction}

The physics of neutron stars represents a way to test our understanding of matter under extreme conditions of density and temperature. Large variation of the density in the range $\sim 10 \times 10^{15} \mathrm{~g} / \mathrm{cm}^{3}$ are indeed expected in neutron stars. This requires the modeling of systems in very different physical conditions such as heavy neutron rich nuclei arranged to form a lattice structure as in the outer crust of the star, or a charge neutral system of interacting hadrons (nucleons, and possibly hyperons or a phase with deconfined quarks) and leptons forming a quantum fluid as in the stellar core [1]. The description of such a variety of systems, of considerable interest for nuclear physics as well as for astrophysics, needs for a challenging theoretical effort and an accurate knowledge of the interactions between the particles present inside the star. The bulk properties of neutron stars (e.g., mass, radius, moment of inertia, mass-shed frequency, tidal deformability, etc.) chiefly depend on the equation of state (EOS) describing the macroscopic properties of stellar matter. The EOS of dense matter is also one of the main ingredients for the study of various astrophysical phenomena related to neutron stars, as core-collapse supernovae (SNe) [2] and binary neutron star (BNS) mergers [3-5]. The recent detection by the LIGO-Virgo collaboration [6] of gravitational waves (GW) from a BNS merger (GW170817) has increased the interest in dense matter physics. Although the structure of a neutron star a few minutes after its birth [1] can be described using a zero temperature EOS, in order to perform realistic numerical simulations for core-collapse SNe and BNS mergers, the inclusion of thermal contributions in the EOS is extremely important. 
In the present work, we model the core of neutron stars as a uniform charge neutral fluid made of neutrons, protons, electrons and muons in equilibrium with respect to the weak interaction ( $\beta$-stable nuclear matter). In addition, we also consider the possible formation of hyperons which are likely to appear in the core of neutron stars due to the very large momenta acquired by nucleons in the hadronic medium [7]. For densities below $0.08 \mathrm{fm}^{-3}$, i.e., to describe the neutron star crust, we employ the EOS reported in Reference [8]. Accordingly we calculate various neutron star properties making use of two EOSs for the stellar core obtained within a microscopic non-relativistic approach based the Brueckner-Bethe-Goldstone (BBG) many-body theory and adopting the Brueckner-Hartree-Fock (BHF) approximation [9]. We also consider some EOSs derived using the relativistic mean field (RMF) approach with the aim of comparing with the results obtained using microscopic EOS models.

\section{Equation of State for Neutron Star Matter}

The main ingredient needed to describe the structure of neutron stars is the EOS namely the relation between the pressure, the energy density and the temperature of the system. In the last 40 years, several approaches have been developed to derive the dense matter EOS. Among non-relativistic approaches, some of the most popular models are based on effective Skyrme interactions which can be used both to describe finite nuclei as well as nuclear matter and neutron stars $[8,10,11]$. In this approach, the parameters of an effective nuclear interaction are adjusted to reproduce the binding energies of nuclei and/or the saturation properties of symmetric nuclear matter. Microscopic many-body approaches such as the BHF [9], the auxiliary-field-diffusion Monte Carlo (AFDMC) [12,13], or the variational Monte Carlo (VMC) [14], start instead from two- and three-body forces fitted to reproduce nucleon-nucleon scattering data and light nuclei properties, and try to derive a parameters free EOS. Finally, RMF approaches [15] are based on effective Lagrangians based on quantum field theory. The Euler-Lagrange equations are solved in mean field approximation adopting the Hartree or Hartree-Fock scheme. The parameters of the effective Lagrangian are usually fixed to reproduce various nuclear matter properties at the empirical saturation density [15]. Each of these approaches has clearly advantages and drawbacks. Non-relativistic many-body approaches for instance have the advantage to start up from the microphysics provided by the nuclear interactions and then they aim to calculate the EOS in a parameter-free way. However non-relativistic approaches do not guarantee the causality condition $v_{s} \leq c$ (with $v_{s}$ the speed of sound in matter and $c$ the speed of light in vacuum) at high baryon density.

In RMF theories, point-like nucleons interact through the exchange of virtual mesons, so that static potentials need not to be introduced. The effects of relativistic propagation are naturally incorporated, so the causality condition is strictly achieved. The coupling constants and some meson masses of the effective meson-nucleon Lagrangian are taken as free parameters, and are adjusted to reproduce the properties of finite nuclei and of nuclear matter at the empirical saturation density.

In the present work, we use two different microscopic EOS models for $\beta$-stable nuclear matter derived within the BHF approach starting from two different non-relativistic Hamiltonians containing two-body and three-body nuclear interactions. As it is well known, three-nucleon forces (TNFs) play a very important role in nuclear physics. For example, TNFs are required to reproduce the experimental binding energy of few-nucleon $(A=3,4)$ systems [16]. TNFs are also essential to reproduce the empirical saturation point $\left(n_{0}=0.16 \pm 0.01 \mathrm{fm}^{-3}, E /\left.A\right|_{n_{0}}=-16.0 \pm 1.0 \mathrm{MeV}\right)$ of symmetric nuclear matter [17-20], and to give an adequately stiff EOS [21-24] which is consistent with present measured neutron star masses and in particular with the mass $M=2.01 \pm 0.04 M_{\odot}$ [25] of the neutron star in PSR J0348 + 0432.

A modern approach, based on a seminal idea of Weinberg [26], to derive two- as well as many-body nuclear interactions is the one provided by chiral effective field theory (ChEFT) (for a review, see Reference $[27,28]$ ). In this method two-, three- and many-body nuclear interactions can be calculated order by order according to a well defined procedure based on a low-energy effective 
quantum chromodynamics (QCD) Lagrangian. This Lagrangian is built in such a way to keep the main symmetries of QCD and in particular the approximate chiral symmetry.

The first two EOSs considered in the present work differ in the two- and three-body interactions used as input. In the first case, we have employed interactions derived in the so-called $\Delta$-full chiral effective field theory in both the two- and three-nucleon sectors, as reported in Reference [21]. These interactions provide very good saturation properties of nuclear matter, as discussed in a previous low density study [18]. This EOS model (hereafter the BL EOS) has been recently used in Reference [29] to simulate the merging of two neutron stars. A finite temperature version of the BL EOS model is under development.

The second microscopic EOS model has been derived within the BHF approach using very simple two- and three-nucleon potentials calculated at leading order (LO) of ChEFT and imposing additional constraints set by the unitary limit of the nucleon-nucleon system [30]. The EOS models of Reference [30] have the remarkable feature that allow for a combined description of nuclear matter saturation properties as well as few-body nuclear systems such as the ${ }^{3} \mathrm{H},{ }^{3} \mathrm{He}$ and ${ }^{4} \mathrm{He}$ nuclei. In the present calculations, we have used the interaction parameters set $\beta=5 \mathrm{fm} ; r_{3}=1.25 \mathrm{fm}$ in Table 1 of Reference [30] (hereafter the LO EOS).

Table 1. Maximum mass configuration properties for the EOS models (first column) considered in this work. In the other columns are reported, respectively: the stellar gravitational maximum mass $M_{\max }$, the corresponding radius $R$, the central baryon number density $n_{c}$ and the central energy density $\epsilon_{c}$. Stellar masses are given in units of the solar mass: $M_{\odot}=1.989 \times 10^{33} \mathrm{~g}$.

\begin{tabular}{ccccc}
\hline Model & $\boldsymbol{M}_{\boldsymbol{m a x}}\left[\boldsymbol{M}_{\odot}\right]$ & $\mathbf{R}[\mathbf{k m}]$ & $\boldsymbol{n}_{\boldsymbol{c}}\left[\mathrm{fm}^{-3}\right]$ & $\boldsymbol{\varepsilon}_{\boldsymbol{c}}\left[\mathbf{M e V f m}^{-3}\right]$ \\
\hline BL & 2.08 & 10.26 & 1.156 & 1535 \\
LO & 2.52 & 11.65 & 0.842 & 1129 \\
TM1-2 & 2.23 & 12.17 & 0.858 & 1064 \\
TM1-2Y & 1.98 & 12.23 & 0.877 & 1042 \\
GM3 & 2.01 & 10.97 & 1.088 & 1393 \\
\hline
\end{tabular}

We also consider three different EOS models based on the RMF approach. In particular, we use the so-called TM1-2 EOS (see Reference [31]) considering both the cases in which hyperons are included (TM1-2Y) or not (TM1-2), and the GM3 parametrization of the Glendenning-Moszkowski model [15] which is a pure nucleonic model. Notice that in the present work we do not consider the possibility of a transition to a phase with deconfined quarks (quark matter) in neutron star matter [15,32-41], even though the formation of quark matter could be expected due to the large densities reached at the center of the most massive neutron stars.

\section{Results}

In Figure 1, we show the total (i.e., including the leptonic contribution) pressure $P$ as a function of the total energy density $\varepsilon$ for $\beta$-stable matter for all the EOS models considered in the present work. Even though all the considered EOSs reproduce (within the experimental uncertainties) nuclear matter properties at the saturation density $n_{0}$, as expected, their predictions are substantially different in the high density regime. With these EOS models as an input, we next numerically integrate the Tolman-Oppenheimer-Volkoff (TOV) equation for hydrostatic equilibrium in general relativity [42,43] to get the structural properties of non-rotating neutron stars. The solutions of these equations are reported in Figure 2 where we plot the (gravitational) mass $M$ of the star as a function of its radius $R$ (Figure 2, left) and the mass $M$ versus the central baryonic density $n_{c}$ (Figure 2, right) for the considered EOSs. The strip with boundaries marked with orange lines stands for the measured mass $M=2.01 \pm 0.04 M_{\odot}[25]$ of the neutron stars in PSR J0348 + 0432. All the models that we have employed are consistent with such measurement. Various structural properties of the maximum mass configuration for the considered EOS models are listed in Table 1. 


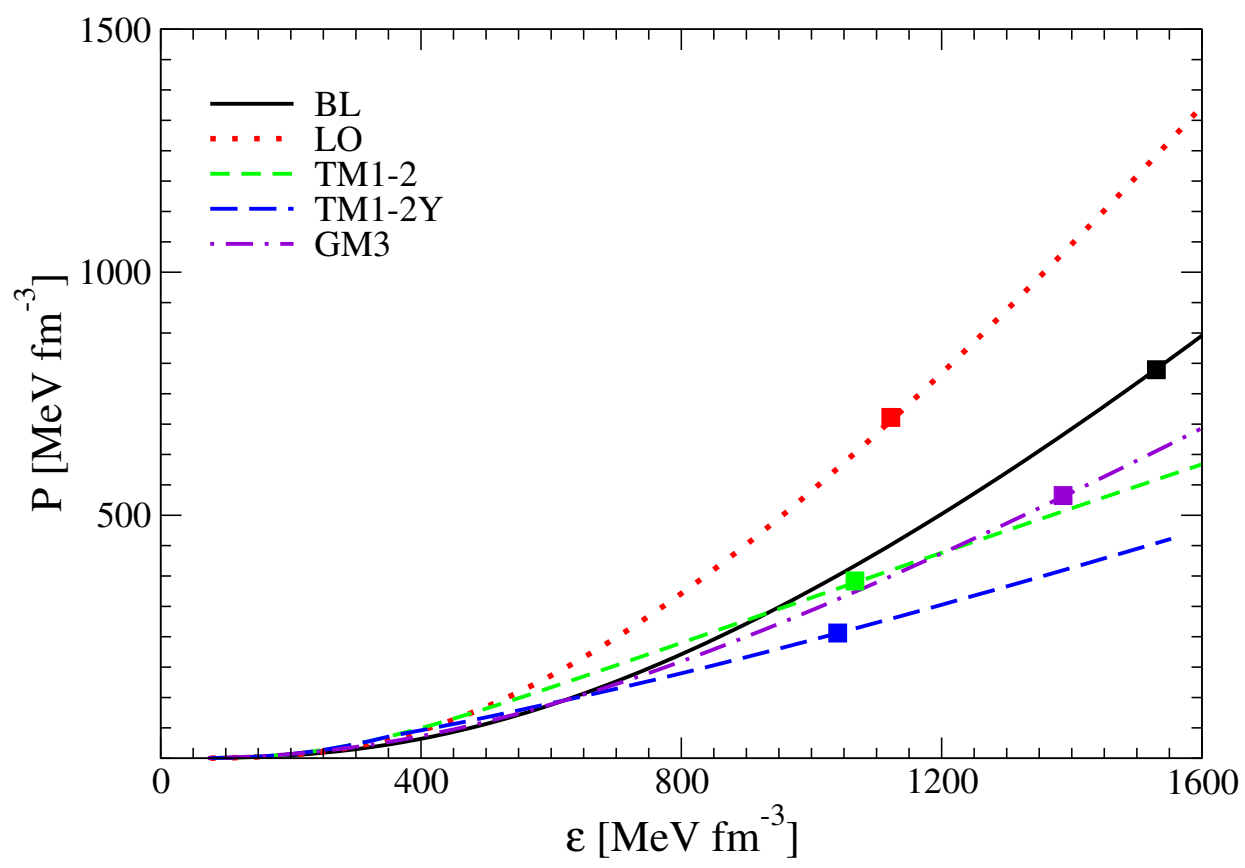

Figure 1. Equation of state for the models described in the text. $P$ denotes the total (i.e., including the leptonic contribution) pressure and $\varepsilon$ the total energy density for $\beta$-stable matter. The square on each curve marks the value of the central energy density $\left(\varepsilon_{c}\right)$ of the neutron star maximum mass configuration for the corresponding EOS model (see fifth column in Table 1).
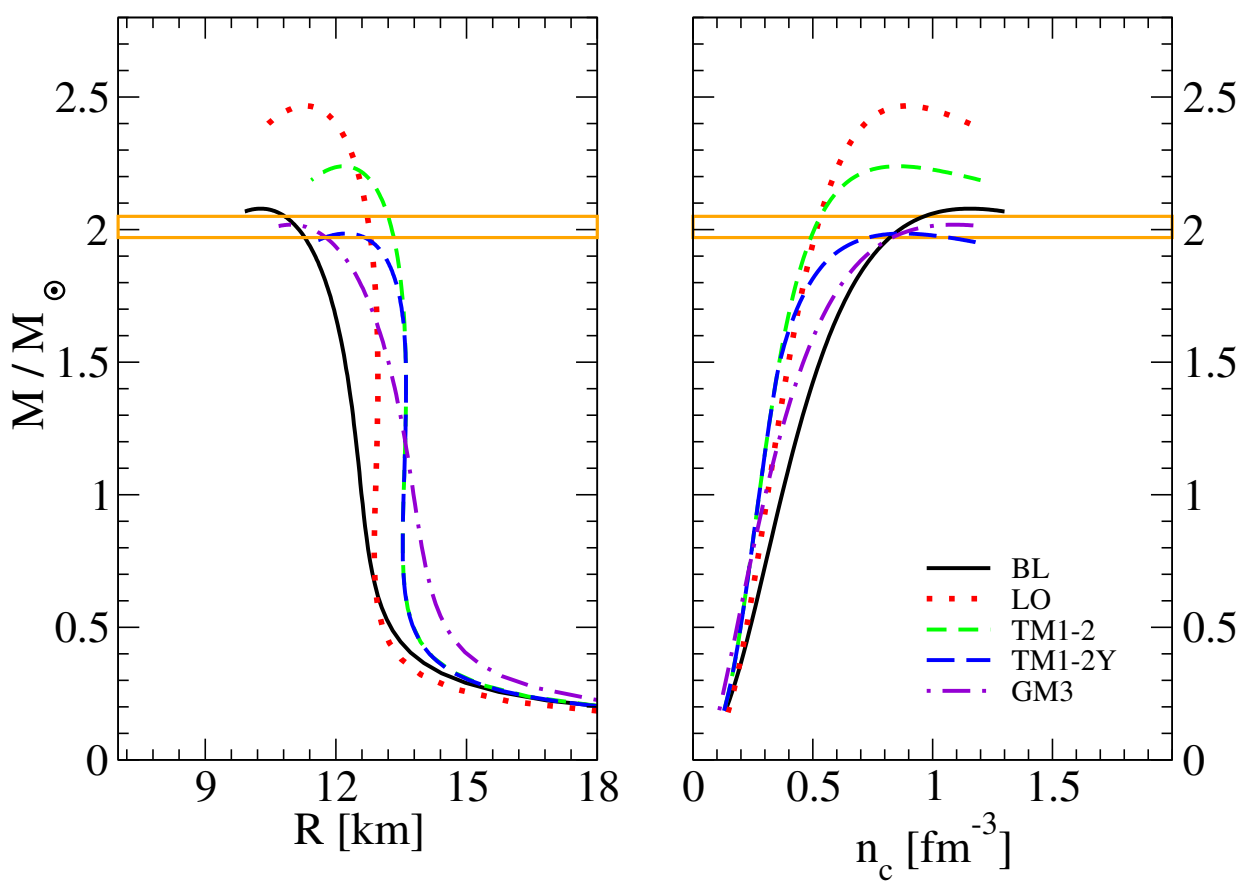

Figure 2. Mass-radius relationship (left) and mass-central baryon density relationship (right) for the EOS models considered in this work. The strip with boundaries marked with orange lines stands for the measured mass $M=2.01 \pm 0.04 M_{\odot}$ [25] of the neutron stars in PSR J0348 +0432 .

As it is well known, to obtain much tighter constraints on the dense matter EOS, with respect to those derived from accurate mass measurements of "heavy" $\left(M \sim 2 M_{\odot}\right)$ neutron stars, it would be highly valuable to get combined measurements of the mass and radius for a certain number of neutron stars. These measurements will be very soon provided by the Neutron star Interior Composition 
ExploreR (NICER) X-ray telescope [44] and, in a not-too-distant future, by the Enhanced X-ray Timing and Polarimetry (eXTP) mission [45] expected to be launched in the mid-2020s.

GW170817 gives a new opportunity to get combined constraints of the mass and radius of the binary's component neutron stars [6] and thus to probe the dense matter EOS. During the inspiral phase of the coalescence, the influence of the EOS enters via the mutual tidal effects of the two stars.

A static, spherically symmetric star of mass $M$ placed in a static external quadrupolar tidal field $\varepsilon_{i j}$ will develop in response an induced quadrupole moment $Q_{i j}$, which is given (to linear order in $\varepsilon_{i j}$ ) by $Q_{i j}=\lambda \varepsilon_{i j}$, where $\lambda$ is called tidal deformability (polarizability) of the star. The parameter $\lambda$ can be written in terms of the stellar radius $R$ and of the tidal Love number $k_{2}$ as:

$$
\lambda=\frac{2}{3} \frac{k_{2}}{G} R^{5}
$$

where $G$ is the gravitational constant. The (quadrupolar) tidal Love number $k_{2}$, which is a dimensionless quantity, depends on the mass of the star and on the EoS of stellar matter and in the case of relativistic stars can be calculated as described in [46-50] .

It is customary to introduce the dimensionless tidal deformability:

$$
\Lambda=\frac{2}{3} k_{2}\left(\frac{c^{2} R}{G M}\right)^{5}
$$

where the tidal deformation of the star is expressed in terms of the stellar compactness $\beta=G M /\left(c^{2} R\right)$.

The tidal effects between the two neutron stars cause the phase of the GW signal to differ from that emitted in the inspiral of point-mass bodies [48,49]. This phase change in the GW signal has been measured in the case of GW170817 [6] and to leading order in post-Newtonian theory is related to the dimensionless binary tidal deformability:

$$
\tilde{\Lambda}=\frac{16}{13}\left[\frac{\left(M_{1}+12 M_{2}\right) M_{1}^{4} \Lambda_{1}+\left(M_{2}+12 M_{1}\right) M_{2}^{4} \Lambda_{2}}{\left(M_{1}+M_{2}\right)^{5}}\right],
$$

where $M_{1}\left(M_{2}\right)$ and $\Lambda_{1}\left(\Lambda_{2}\right)$ are the mass and tidal deformability for the high (low) mass component of the binary system.

A new analysis (with respect to the original one [6]) of the GW170817 data has been carried out in References [51-53]. In particular, as shown in Reference [53], such analysis allowed obtaining a robust constraint on the nuclear matter EOS up to $\sim 2 n_{0}$. Remarkably, similar results have been obtained in the independent analysis performed in Reference [54]. Future detections may provide the opportunity to improve and extend such constraints on the EOS to higher nuclear densities.

In Figure 3, we report the posterior probability density function for the tidal deformability parameters $\Lambda_{1}$ and $\Lambda_{2}$ for the two merging neutron stars inferred from the detected signals in GW170817 derived in Reference [53]. The dark green, brown, and orange lines, taken from Figure 1 of Reference [53], denote 50\% (dashed) and 90\% (solid) credible levels for the posteriors obtained using EOS-insensitive relations, a parametrized EOS without a maximum mass requirement, and independent EOSs [51]. The gray shading corresponds to the unphysical region where $\Lambda_{2}<\Lambda_{1}$. The deduced constraints on the tidal deformabilities depend on assumptions on the dimensionless neutron star spin $\chi \equiv c J /\left(G M^{2}\right)$ (where $J$ is the angular momentum of the neutron star) for the two components. Here, we consider only the "low-spin" prior analysis which assumes $\chi<0.05$. This upper value for $\chi$ is compatible with measured pulsar spins in Galactic double neutron star binaries. In addition, for the "low-spin" prior case, the lower bound value, $M_{2}=1.18 M_{\odot}$ (see Table III in Reference [52]) for the low-mass component $\left(M_{2} \leq M_{1}\right)$, is still compatible with measured neutron star masses, and in particular with the measured mass $M=1.174 \pm 0.004 M_{\odot}$ for the companion star in the double neutron star system PSR J0453 + 1559 [55]. In the same figure, we show the tidal deformabilities $\Lambda_{2}$ and $\Lambda_{1}$ for the two binary components, calculated as described in [47-50], for the EOS models 
considered in the present work. The portion of the curve corresponding to each EOS model is obtained by varying the value of the mass of the high-mass component in the range $1.36 \leq M_{1} / M_{\odot} \leq 1.58$, obtained from GW170817 data [52] (for the "low-spin" prior case) and next deriving the value $M_{2}$ for the low-mass component by keeping the chirp mass $M_{\text {chirp }}=\left(M_{1} M_{2}\right)^{3 / 5}\left(M_{1}+M_{2}\right)^{-1 / 5}$ fixed to the measured value $M_{\text {chirp }}=1.186 M_{\odot}$ [52].

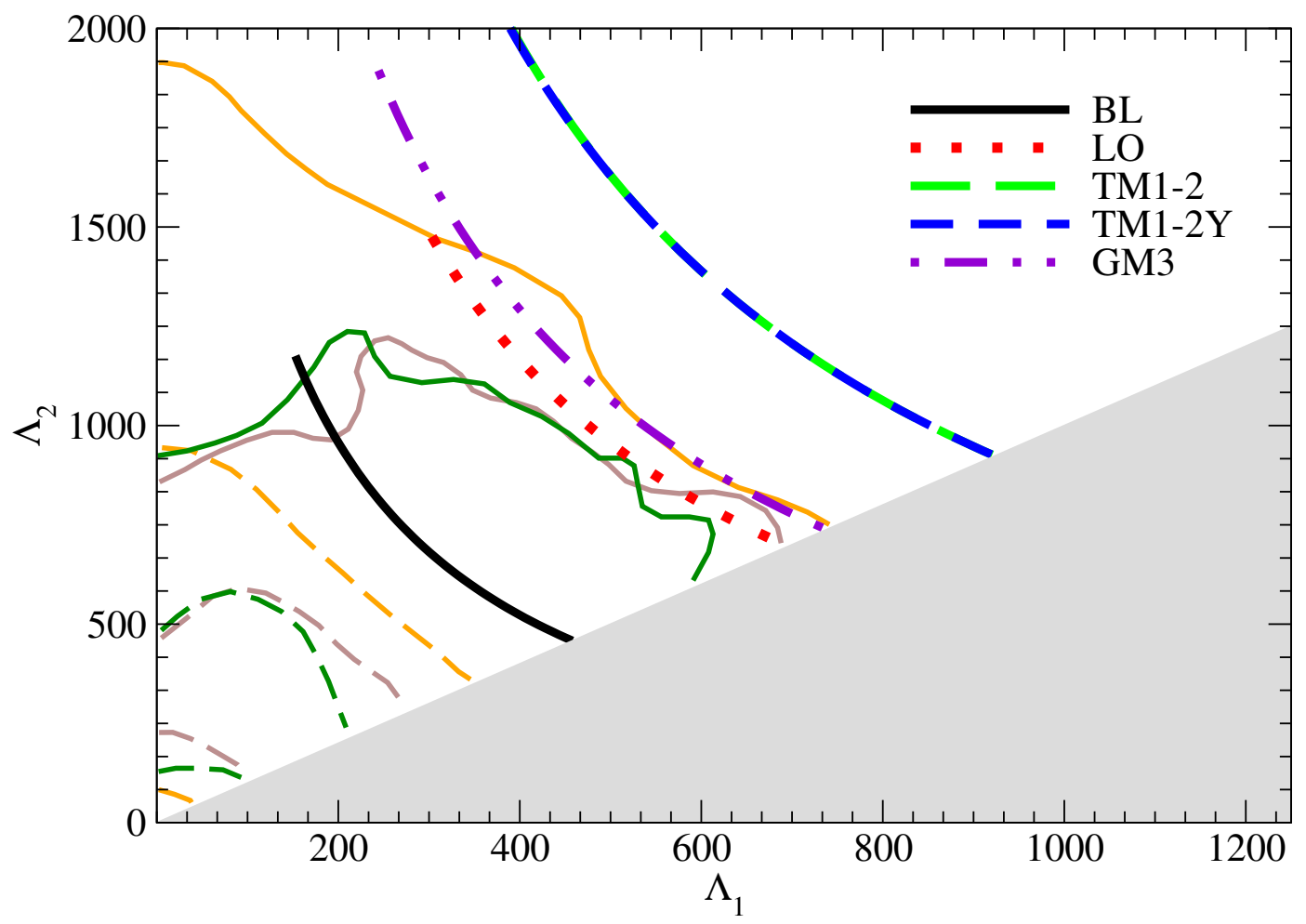

Figure 3. (Color online) Tidal deformabilities $\Lambda_{1}, \Lambda_{2}$ of the two merging neutron stars for the EOS models adopted in the present work. The dark green, brown, and orange lines, taken from Reference [53], denote 50\% (dashed) and 90\% (solid) credible levels for the posteriors obtained using EOS-insensitive relations, a parameterized EOS without a maximum mass requirement, and independent EOSs [51]. The gray shading corresponds to the unphysical region where $\Lambda_{2}<\Lambda_{1}$. Here, we consider "low-spin" prior $|\chi| \leq 0.05$ for the adimensional spins of the two neutron stars.

The BL EOS is compatible with the $90 \%$ credible region for $\Lambda_{1}$ and $\Lambda_{2}$ obtained from GW170817 data while the LO model is within the $90 \%$ credible region only considering the case of independent EOSs [53]. It is interesting to observe that the fully microscopic models $\mathrm{BL}$ and LO, which have been obtained using interactions derived in ChEFT, are able to provide a good description of data analysis of GW170817. The GM3 model is just partially consistent with the constraints obtained in the case of independent EOSs. Finally, the tidal deformabilities for the models TM1 and TM1-2Y lie outside the $90 \%$ of credible region, thus these EOS models are unfavored with respect to the microscopic BL and LO EOS models. Notice that the two curves in Figure 3 relative to the TM1-2 (nucleons only) EOS and to the TM1-2Y (nucleons plus hyperons) EOS are one on the top of the other. This result is not surprising in view of the following considerations. For the considered parameterization of the TM1-2Y model, the hyperon threshold is equal to $\sim 0.35 \mathrm{fm}^{-3}$, which corresponds to a neutron star with $\mathrm{M} \sim 1.36 M_{\odot}$. We note that for the high mass component $M_{1}$ of the GW170817 binary system, even considering the upper value $M_{1}=1.58 M_{\odot}$ [52,53], the total hyperons number density at the center of this star is just $\sim 4 \%$ of the total baryon number density. Thus, within the TM1-2Y model, hyperons are barely present in the binary's component neutron stars in GW170817 during their inspiral phase. Larger hyperons concentrations could be present in the post-merger compact object and show peculiar signatures in the GW signal at a few $\mathrm{kHz}[56,57]$, which could be detected with the third 
generation of GW observatories. A similar analysis can be extended also to the case of the formation of other degrees of freedom which may be produced in the high density core of neutron stars such as quark matter or meson condensates [33,58].

A very interesting consequence of GW detection in GW170817 has been the possibility to translate the informations on the tidal polarizability on constraints on the neutron star radius [53]. This has been done by different research groups using different assumptions and strategies. Assuming that the two merging neutron stars obey the same EOS, which was not imposed in the initial LIGOVirgo analysis [6], the authors of Reference [54] found $\tilde{\Lambda}=245_{-151}^{+453}$ at $90 \%$ of confidence level and a common radius of $R=10.9_{-1.6}^{+2.1} \pm 0.2 \mathrm{~km}$ for a $1.4 M_{\odot}$ neutron star. A similar parameter estimation of GW170817 has been proposed in Reference [59] where, in addition to GW data, data from electromagnetic counterparts have been included. The authors of Reference [59] found: $\tilde{\Lambda}$ in the interval $(323 ; 776)$ at $90 \%$ of confidence level and common neutron star radius $R=12.2_{-0.8}^{+1.0} \pm 0.2 \mathrm{~km}$. Finally, in Reference [60], the GW data were combined with radiative transfer models of the kilonova lightcurve, phenomenological fits from numerical relativity simulations of the disk and dynamical ejecta masses, and fits between the GRB jet energy and disk mass. These authors found: $\tilde{\Lambda}$ in the interval $(292 ; 822)$ and common neutron star radius in the range $(11.1 ; 13.4) \mathrm{km}$. We note that, although the findings of Reference [60] are in agreement with the predictions of References [54,59], they are based on the strong assumption about the electromagnetic model fitting. Note that the prediction of the radius for a 1.4 $M_{\odot}$ neutron star for the BL and LO models (see Figure 2 ) is in very good agreement with that reported in the above quoted papers $[54,59,60]$. The models based on the RMF approach, at least those used in the present paper, show instead larger radii than those inferred from GW170817 [53,54,59,60]. In the future, when hopefully a large database of GW data coming from neutron star merging will be created, it may be possible to strongly constrain the EOS of neutron star matter and even to get informations on the nuclear interactions in the medium.

\section{Conclusions}

In the present work, we have studied how recent estimates of the tidal deformabilities of the binary's component neutron stars, obtained by the analysis of the GW data in the event GW170817, can help to constrain the EOS of neutron star matter. To this end, we have considered some microscopic and phenomenological EOS models for nucleonic as well as hyperonic matter. All the considered EOS models reproduce, within the experimental uncertainties, empirical nuclear matter properties at the saturation density $n_{0}$ and are consistent with present measured neutron star masses. For each of these models, we have determined various neutron star structural properties and calculated the tidal deformabilities $\Lambda_{1}$ and $\Lambda_{2}$ for the two merging neutron stars. We have found that the microscopic EOS model BL produces a very good agreement both considering the posterior distribution obtained from the data analysis of GW170817 and the mass-radius constraints on a $1.4 M_{\odot}$ neutron star obtained by different research groups $[54,59,60]$. Using GW170817 data and within the TM1-2Y EOS model, we cannot make any significant statement about the possible presence of hyperons in the two stars during their inspiral phase. Hyperons could be abundantly present in the post-merger object and strongly influence its evolution. Large hyperon fractions in the post-merger object can give peculiar signatures in the GW signal at a few $\mathrm{kHz}$, which could be detected with the third generation of GW observatories.

Author Contributions: Conceptualization and methodology, D.L. and I.B.; software, D.L. and I.B.; investigation, D.L. and I.B.; writing-original draft preparation, D.L. and I.B.; writing-review and editing, D.L. and I.B.; visualization, D.L. and I.B.; supervision, D.L. and I.B.; project administration, D.L. and I.B.; and funding acquisition, D.L. and I.B.

Acknowledgments: We thank one of the referees for useful suggestions that improved the quality of the paper. We thank Rosa Poggiani for useful discussions about GW170817 data analysis. This work has been supported by "PHAROS: The multi-messenger physics and astrophysics of compact stars", COST Action CA16214.

Conflicts of Interest: The authors declare no conflict of interest. 


\section{References}

1. Prakash, M.; Bombaci, I.; Prakash, M.; Ellis, P.J.; Lattimer, J.M.; Knorren, R. Composition and structure of protoneutron stars. Phys. Rep. 1997, 280, 1. [CrossRef]

2. Oertel, M.; Hempel, M.; Klahn, T.; Typel, S. Equations of state for supernovae and compact stars. Rev. Mod. Phys. 2017, 89, 015007. [CrossRef]

3. Bernuzzi, S.; Dietrich, T.; Nagar, A. Modeling the complete gravitational wave spectrum of neutron star mergers. Phys. Rev. Lett. 2015, 115, 091101. [CrossRef] [PubMed]

4. Sekiguchi, Y.; Kiuchi, K.; Kyutoku, K.; Shibata, M.; Taniguchi, K. Dynamical mass ejection from the merger of asymmetric binary neutron stars: Radiation-hydrodynamics study in general relativity. Phys. Rev. D 2016, 93, 124046. [CrossRef]

5. Baiotti, L.; Rezzolla, L. Binary neutron star mergers: A review of Einstein's richest laboratory. Rep. Prog. Phys. 2017, 80, 9. [CrossRef] [PubMed]

6. Abbott, B.P.; et al. [LIGO Scientific Collaboration and Virgo Collaboration] GW170817: Observation of gravitational waves from a binary neutron star inspiral. Phys. Rev. Lett. 2017, 119, 161101. [CrossRef] [PubMed]

7. Vidaña, I.; Logoteta, D.; Providência, C.; Polls A.; Bombaci, I. Estimation of the effect of hyperonic three-body forces on the maximum mass of neutron stars. Europhys. Lett. 2011, 94, 11002. [CrossRef]

8. Douchin, F.; Haensel, P. A unified equation of state of dense matter and neutron star structure. Astron. Astrophys. 2001, 380, 151. [CrossRef]

9. Baldo, M.; Burgio, G.F. Properties of the nuclear medium. Rep. Progr. Phys. 2012, 75, 026301. [CrossRef] [PubMed]

10. Dutra, M.; Lourenço, O.; Martins, J.S.; Delfino, A.; Stone, J.R.; Stevenson, P.D. Skyrme interaction and nuclear matter constraints. Phys. Rev. C 2012, 85, 035201. [CrossRef]

11. Lattimer, J.M.; Swesty, D.F. A generalized equation of state for hot, dense matter. Nucl. Phys. 1991, 535, 331. [CrossRef]

12. Schmidt, K.E.; Fantoni, S. A quantum Monte Carlo method for nucleon systems. Phys. Lett. B 1999, 446, 99. [CrossRef]

13. Lonardoni, D.; Gandolfi, S.; Lynn, J.E.; Petrie, C.; Carlson, J.; Schmidt, K.E.; Schwenk, A. Auxiliary field diffusion Monte Carlo calculations of light and medium-mass nuclei with local chiral interactions. Phys. Rev. Lett. 2018, 97, 044318. [CrossRef]

14. Carlson, J.; Gandolfi, S.; Pederiva, F.; Pieper, S.C.; Schiavilla, R.; Schmidt, K.E.; Wiringa, R.B. Quantum Monte Carlo methods for nuclear physics. Rev. Mod. Phys. 2015, 87, 1067. [CrossRef]

15. Glendenning, N.K. (Ed.) Compact Stars: Nuclear Physics, Particle Physics, and General Relativity; Springer: New York, NY, USA, 2000.

16. Kalantar-Nayestanaki, N.; Epelbaum, E.; Messchendorp, J.S.; Nogga, A. Signatures of three-nucleon interactions in few-nucleon systems. Rep. Prog. Phys. 2012, 75, 016301. [CrossRef] [PubMed]

17. Logoteta, D.; Vidaña, I.; Bombaci, I.; Kievsky, A. Comparative study of three-nucleon force models in nuclear matter. Phys. Rev. C 2015, 91, 064001. [CrossRef]

18. Logoteta, I.; Bombaci, I.; Kievsky, A. Nuclear matter saturation with chiral three-nucleon interactions fitted to light nuclei properties. Phys. Lett. B 2016, 758, 449. [CrossRef]

19. Logoteta, I.; Bombaci, I.; Kievsky, A. Nuclear matter properties from local chiral interactions with $\Delta$ isobar intermediate states. Phys. Rev. C 2016, 94, 064001. [CrossRef]

20. Logoteta, I. Optimized chiral N2LO interactions in nuclear matter. Eur. Phys. J. A 2018, 54, 111. [CrossRef]

21. Bombaci, I.; Logoteta, I. Equation of state of dense nuclear matter and neutron star structure from nuclear chiral interactions. Astron. Astrophys. 2018, 609, A128. [CrossRef]

22. Logoteta, I.; Bombaci, I. Neutron star properties from optimized chiral nuclear interactions. Publi. Astron. Soc. Aust. 2018, 35, e035. [CrossRef]

23. Akmal, A.; Pandharipande V.R.; Ravenhall D.G. Equation of state of nucleon matter and neutron star structure. Phys. Rev. C 1998, 58, 1804. [CrossRef]

24. Baldo, M.; Bombaci, I.; Burgio, G.F. Microscopic nuclear equation of state with three-body forces and neutron star structure. Astron. Astrophys. 1997, 328, 274. 
25. Antoniadis, J.; Freire, P.C.; Wex, N.; Tauris, T.M.; Lynch, R.S.; van Kerkwijk, M.H.; Kramer, M.; Bassa, C.; Dhillon, V.S.; Driebe, T.; et al. A massive pulsar in a compact relativistic binary. Science 2012, 340, 1233232. [CrossRef] [PubMed]

26. Weinberg, S. Phenomenological lagrangians. Physica A 1979, 96, 327. [CrossRef]

27. Epelbaum, E.; Hammer, H.W.; Meissner, U.G. Modern theory of nuclear forces. Rev. Mod. Phys. 2009, 81, 1773. [CrossRef]

28. Machleidt, R.; Entem, D.R. Chiral effective field theory and nuclear forces. Phys. Rep. 2011, 501, 1. [CrossRef]

29. Endrizzi, A.; Logoteta, D.; Giacomazzo, B.; Bombaci, I.; Ciolfi, R.; Kastaun, W. Effects of chiral effective field theory equation of state on binary neutron star mergers. Phys. Rev. D 2018, 98, 043015. [CrossRef]

30. Kievsky, A.; Viviani, M.; Logoteta, D.; Bombaci, I.; Girlanda, L. Correlations imposed by the unitary limit between few-nucleon systems and compact stellar systems. Phys. Rev. Lett. 2018, 121, 072701. [CrossRef]

31. Providência, C.; Rabhi, A. Interplay between the symmetry energy and the strangeness content of neutron stars. Phys. Rev. C 2013, 87, 055801. [CrossRef]

32. Marczenko, M.; Blaschke, D.; Redlich, K.; Sasaki, C. Chiral symmetry restoration by parity doubling and the structure of neutron stars. Phys. Rev. D 2018, 98, 103021. [CrossRef]

33. Bombaci, I.; Logoteta, D.; Vidaña, I.; Providência, C. Quark matter nucleation in neutron stars and astrophysical implications. Eur. Phys. J. A 2016, 52, 58. [CrossRef]

34. Bombaci, I.; Logoteta, I. A link between measured neutron star masses and lattice QCD data. Mon. Not. R. Astron. Soc. Lett. 2013, L79, 433. [CrossRef]

35. Logoteta, I.; Bombaci, I. Quark deconfinement transition in neutron stars with the field correlator method. Phys. Rev. D 2013, 88, 063001. [CrossRef]

36. Drago, A.; Lavagno, A.; Pagliara, G. Effects of color superconductivity on the structure and formation of compact stars. Phys. Rev. D 2004, 69, 057505. [CrossRef]

37. Lugones, G.; Bombaci, I. Deconfinement and color superconductivity in cold neutron stars. Phys. Rev. D 2005, 72, 065021. [CrossRef]

38. Logoteta, D.; Bombaci, I.; Providência, C.; Vidaña, I. Quark matter nucleation with a microscopic hadronic equation of state. Phys. Rev. C 2012, 85, 055807. [CrossRef]

39. Dexheimer, V.; Steinheimer, J.; Negreiros, R.; Schramm, S. Hybrid stars in an SU(3) parity doublet model. Phys. Rev. C 2013, 87, 015804. [CrossRef]

40. Logoteta, D.; Bombaci, I.; Providência, C.; Vidaña, I. Chiral model approach to quark matter nucleation in neutron stars. Phys. Rev. D 2012, 85, 023003. [CrossRef]

41. Logoteta, D. Constraints on hybrid neutron stars equation of state from neutron stars merging. Eur. Phys. J. A 2019, 55, 133. [CrossRef]

42. Tolman, R.C. (Ed.) Relativity, Thermodynamica and Cosmology; Oxford University Press: Oxford, UK, 1934.

43. Oppenheimer, J.; Volkoff, G. On Massive Neutron Cores. Phys. Rev. 1939, 55, 374. [CrossRef]

44. Gendreau, K.C.; Arzoumanian, Z.; Adkins, P.W.; Albert, C.L.; Anders, J.F.; Aylward, A.T.; Baker, C.L.; Balsamo, E.R.; Bamford, W.A.; Benegalrao, S.S.; et al. The Neutron star Interior Composition Explorer (NICER): Design and development. In Proceedings of the Space Telescopes and Instrumentation 2016: Ultraviolet to Gamma Ray, Edinburgh, UK, 26 June-1 July, 2016; p. 99051H.

45. Watts, A.L.; Yu, W.; Poutanen, J.; Zhang, S.; Bhattacharyya, S.; Bogdanov, S.; Ji, L.; Patruno, A.; Riley, T.E.; Bakala, P.; et al. Dense matter with eXTP. Sci. China Phys. Mech. Astron. 2019, 62, 29503. [CrossRef]

46. Damour, T. Gravitational radiation and the motion of compact bodies. In Gravitational Radiation; Deruelle, N., Piran, T., Eds.; North-Holland: Amsterdam, The Netherlands, 1983; pp. 59-144.

47. Hinderer, T. Tidal Love numbers of neutron stars. Astrophys. J. 2008, 677, 1216. [CrossRef]

48. Damour, T.; Nagar, A. Relativistic tidal properties of neutron stars. Phys. Rev. D 2009, 80, 084035. [CrossRef]

49. Damour, T.; Nagar, A.; Villain, L. Measurability of the tidal polarizability of neutron stars in late-inspiral gravitational-wave signals. Phys. Rev. D 2012, 85, 123007. [CrossRef]

50. Postnikov, S.; Prakash, M.; Lattimer, J.M. Tidal Love numbers of neutron and self-bound quark stars. Phys. Rev. D 2010, 82, 024016. [CrossRef]

51. Abbott, B.P.; et al. [LIGO Scientific Collaboration and Virgo Collaboration] Properties of the Binary Neutron Star Merger GW170817. Phys. Rev. X 2019, 9, 011001. 
52. Abbott, B.P.; et al. [LIGO Scientific Collaboration and Virgo Collaboration] GWTC-1: A gravitational-wave transient catalog of compact binary mergers observed by LIGO and Virgo during the First and Second Observing Runs. Phys. Rev. X 2019, 9, 031040.

53. Abbott, B.P.; et al. [LIGO Scientific Collaboration and Virgo Collaboration] GW170817: Measurements of neutron star radii and equation of state. Phys. Rev. Lett. 2018, 121, 161101. [CrossRef]

54. De, S.; Finstad, D.; Lattimer, J.; Brown, D.A.; Berger, E.; Biwer, C.M. Tidal deformabilities and radii of neutron stars from the observation of GW170817. Phys. Rev. Lett. 2018, 121, 091102. [CrossRef]

55. Martinez, J.G.; Stovall, K.; Freire, P.C.C.; Deneva, J.S.; Jenet, F.A.; McLaughlin, M.A.; Bagchi, M.; Bates, S.D.; Ridolfi, A. Pulsar J0453 + 1559: A double neutron star system with large mass asymmetry. Astrophys. J. 2015, 812, 143. [CrossRef]

56. Sekiguchi, Y.; Kiuchi, K.; Kyutoku, K.; Shibata, M. Effects of hyperons in binary neutron star mergers. Phys. Rev. Lett. 2011, 107, 211101. [CrossRef] [PubMed]

57. Radice, D.; Bernuzzi, S.; Del Pozzo, W.; Ott, C.; Roberts, L.F. Probing extreme-density matter with gravitational wave observations of binary neutron star merger remnants. Astrophys. J. Lett. 2017, 842, 2. [CrossRef]

58. De Pietri, R.; Drago, A.; Feo, A.; Pagliara, G.; Pasquali, M.; Traversi, S.; Wiktorowicz, G. Merger of Compact Stars in the Two-Families Scenario. Available online: https://arxiv.org/abs/1904.01545 (accessed on 24 September 2019).

59. Radice, D.; Dai, L. Multimessenger parameter estimation of GW170817. Eur. Phys. J. A 2018, 55, 50. [CrossRef]

60. Coughlin, M.W.; Dietrich, T.; Margalit, B.; Metzger, B.D. Multi-Messenger Bayesian Parameter Inference of a Binary Neutron-Star Merger. Available online: https://arxiv.org/abs/1812.04803 (accessed on 24 September 2019).

(C) 2019 by the authors. Licensee MDPI, Basel, Switzerland. This article is an open access article distributed under the terms and conditions of the Creative Commons Attribution (CC BY) license (http:/ / creativecommons.org/licenses/by/4.0/). 